\title{
Ultrasonic wave propagation through porous ceramics at different angles of propagation
}

\author{
Haydar Aygün ${ }^{1}$ \\ Maritime and Technology Faculty, East Park Terrace, Southampton Solent University, Southampton, \\ SO14 0RD, UK
}

Short Title: Wave propagation in ceramics

Keywords: ultrasound, wave propagation, ceramics, Biot theory

\begin{abstract}
:
The anisotropic pore structure and elasticity of cancellous bone cause wave speeds and attenuation in cancellous bone to vary with angle. Comparisons between predictions of a Biot-Allard model allowing for angle-dependent elasticity and angleand-porosity dependent tortuosity and transmission data obtained on water-saturated replica bones at normal and oblique incidence are extended to water saturated porous rigid ceramic at different angles of propagation. It is found that predictions of the variation of transmitted waveforms with angle through porous ceramic are in reasonable agreement with data.
\end{abstract}

\section{Introduction}

Bone essentially has two types of structure, both having the same mineralized collagen composition. Cortical bone may generally be considered to be solid; cancellous bone consists of a complex open-celled porous network of rod- and plate-shaped elements termed trabeculae. Osteoporosis is a bone disease caused by hormonal and biochemical changes. Osteoporosis leads to nearly 9 million fractures annually worldwide [1], and over 300,000 patients present with fragility fractures to hospitals in the UK each year [2]. Direct medical costs from fragility fractures to the UK healthcare economy were estimated at $£ 1.8$ billion in 2000 , with the potential to increase to $£ 2.2$ billion by 2025, and with most of these costs relating to hip fracture care [3].

To improve the prediction of fracture risk by ultrasound it is important to understand the propagation of acoustic waves through rigid porous materials such as cancellous bone. Biot theory has been used extensively to describe the wave propagation in cancellous bone [4-12]. It was specifically developed to describe acoustic wave propagation in fluid-saturated porous elastic media $[13,14]$. Biot theory predicts two compressional waves (fast and slow waves), when the waves

\footnotetext{
${ }^{1}$ Electronic mail: haydar-aygun@hotmail.com
} 
propagating through the solid frame of bone and marrow are in-phase and out-of-phase respectively, and a shear wave. It allows for an arbitrary microstructure, with separate motions considered for the solid elastic framework (bone) and the interspersed fluid (marrow), induced by the ultrasonic wave, and also includes energy loss due to viscous friction between solid (bone) and fluid (marrow).

The anisotropic pore structure and elasticity of cancellous bone cause wave speeds and attenuation in cancellous bone to vary with angle. Aygün et al. [15] have extended previous work on the influence of anisotropic pore structure and elasticity in cancellous bone by developing an anisotropic Biot-Allard model allowing for angle-dependent elasticity, and angle-and-porosity dependent tortuosity. The extreme angle dependence of tortuosity corresponding to the parallel plate microstructure used by Hughes et al. [4] has been replaced by angle-and-porosity dependent tortuosity values based on data for slow wave transmission through air-filled stereolithography (STL) bone replicas [16]. It has been suggested that the anisotropic Biot-Allard model could be used to give further insight into the factors that have the most important influence on the angle dependency of wave speeds and attenuation in cancellous bone. Nevertheless the applicability of Biot-based theories to ultrasonic propagation in bone remains in question given the expected role of scattering which is neglected in the these theories.

Aygün et al. $[17,18]$ have transmitted ultrasonic signals through water saturated stereolithograpical bone replicas in the form of $57 \mathrm{~mm}$ cubes with microstructural dimensions that are 13 times real scale at normal angle and oblique angles. Remarkably, it is found that the expected occurrence of scattering does not cause significant discrepancies between predictions and data at $100 \mathrm{kHz}$ (which would be equivalent to $1.3 \mathrm{MHz}$ in real bone), perhaps as a consequence of the fact that the samples behave as low pass filters.

The aim of this paper is to investigate further ultrasonic wave transmission measurements on porous rigid ceramic immersed in water at $1 \mathrm{MHz}$ as a function of angle of propagation. Predictions of the anisotropic Biot-Allard model allowing for angle-dependent elasticity and angle-and-porosity dependent tortuosity have been compared with measurements made in a fluid (water) filled tank at 1 MHz. 


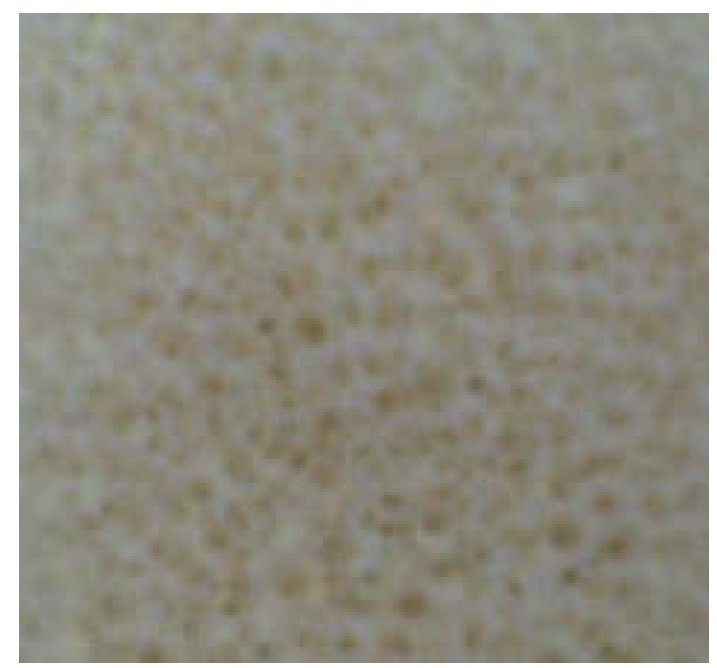

Figure 1: Picture of rigid porous ceramic.

\section{Measurements}

A procedure given by Fellah et al. [8] has been used to carry out measurements on porous rigid ceramic immersed in water filled tank with transducers (see Figure 2). Two broadband Panametrics A $303 \mathrm{~S}$ plane piezoelectric transducers having $1 \mathrm{~cm}$ diameter with $1 \mathrm{MHz}$ central frequency have been used. $400 \mathrm{~V}$ pulses are provided by a 5058PR Panametrics pulser/receiver. Electronic interference is removed by 1000 acquisition averages.

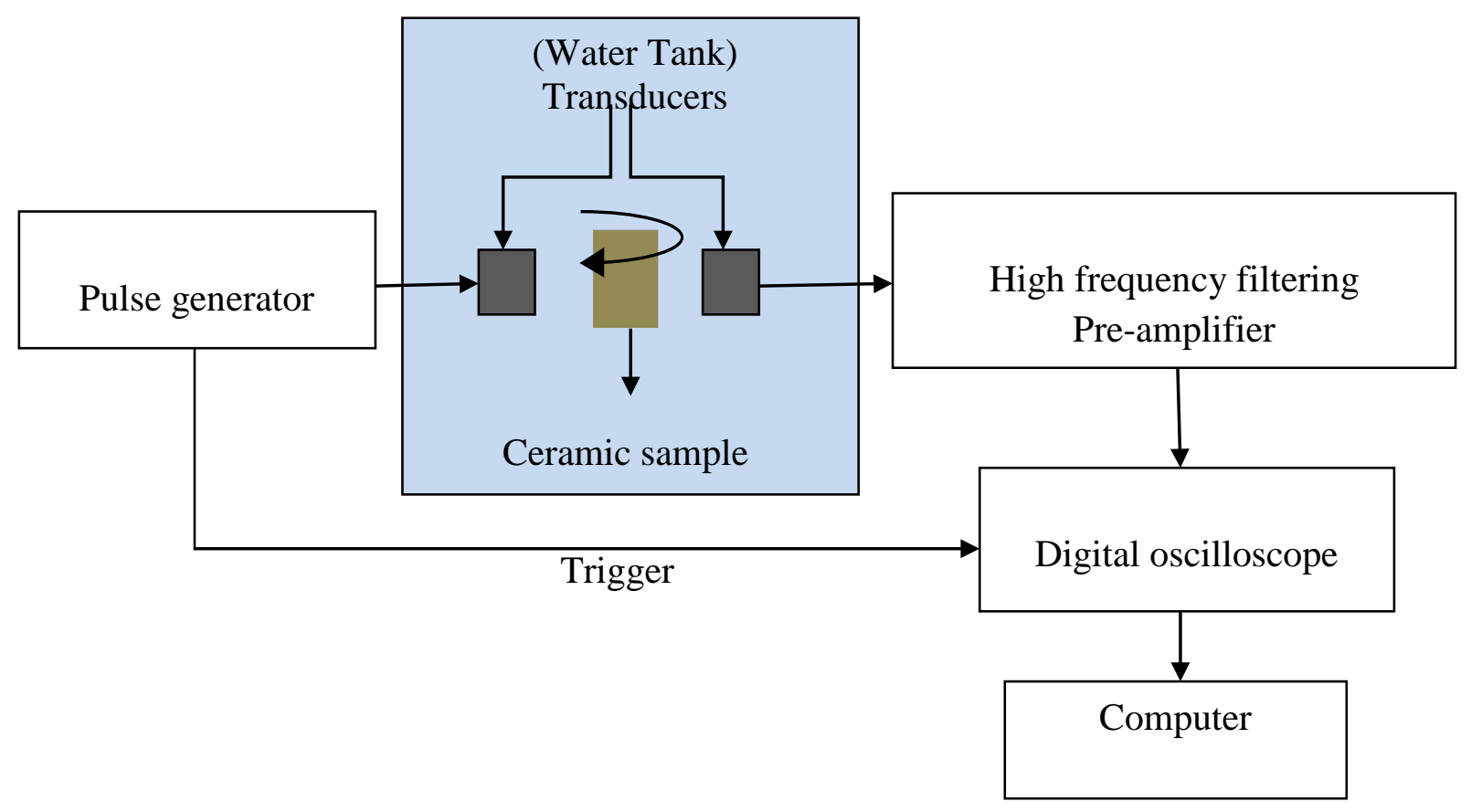

Figure 2: Experimental setup for ultrasonic measurements. 
Porous ceramic is obtained by mixing clay and plastic then burning the plastic in a kiln at Laboratory of Acoustics and Thermal Physics at K. U. Leuven. Porous ceramic used for measurements is in the form of $65 \mathrm{~mm}$ squares with $30 \mathrm{~mm}$ thickness. The measurements have been made parallel to trabeculae direction starting from $0^{0}$ up to $45^{\circ}$. One incident (reference) signal generated by $1 \mathrm{MHz}$ transducers and transmitted over corresponding path lengths in fluid (water) shown in Figure 3a, and its spectra is shown in Figure 3b, respectively. Reference signal was used as an initial signal when analyzing transmission data for porous ceramic.
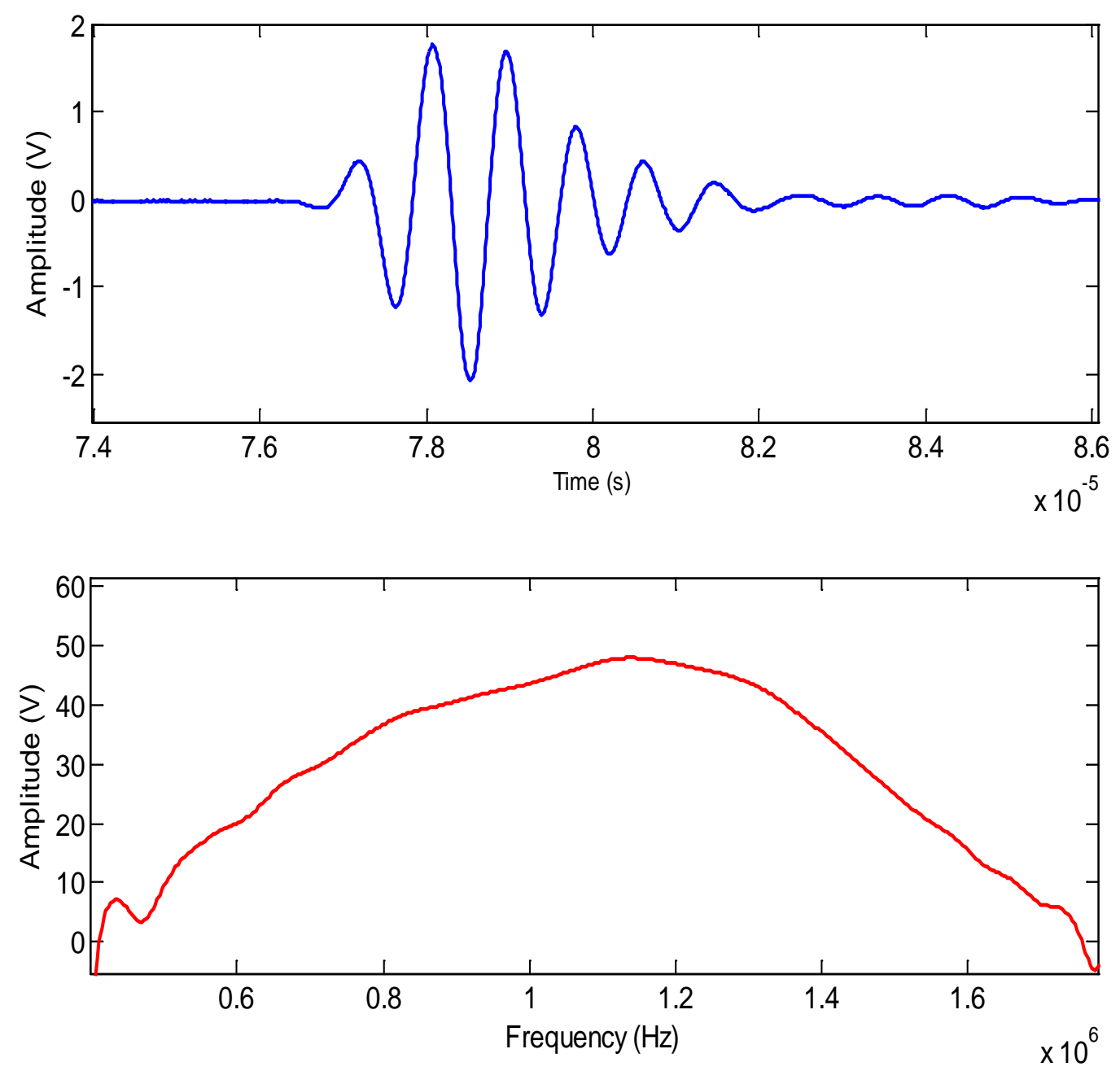

Figure 3: a-) Inicident signal for porous rectangular ceramics versus time, b-) its spectrum versus frequency at $1 \mathrm{MHz}$.

To vary the angle of incidence, ceramic was revolved around its central axis. For rotation angle, $\theta$, measured from the normal, the transmission path becomes $L \cdot \cos (\theta)$ where $L$ is the cube dimension. The distance between two transducers was $115 \mathrm{~mm}$ and this distance was kept same throughout of measurements. Measured variations of transmitted signals through rigid porous ceramic as a function of angle of propagation are shown in Figures 4 and 5. The variation in the signals transmitted through the ceramic is mainly in amplitude rather than in the structure of the waveforms. There is a variation in the amplitude and the structure of the waveform at $30^{\circ}$ (see 
Figure 5). Varying angle of propagation from $0^{0}$ to $45^{0}$ shifts the peak amplitude of waveforms towards right-hand-side because the transmission path length has been increased from $30 \mathrm{~mm}$ to $42.43 \mathrm{~mm}$.

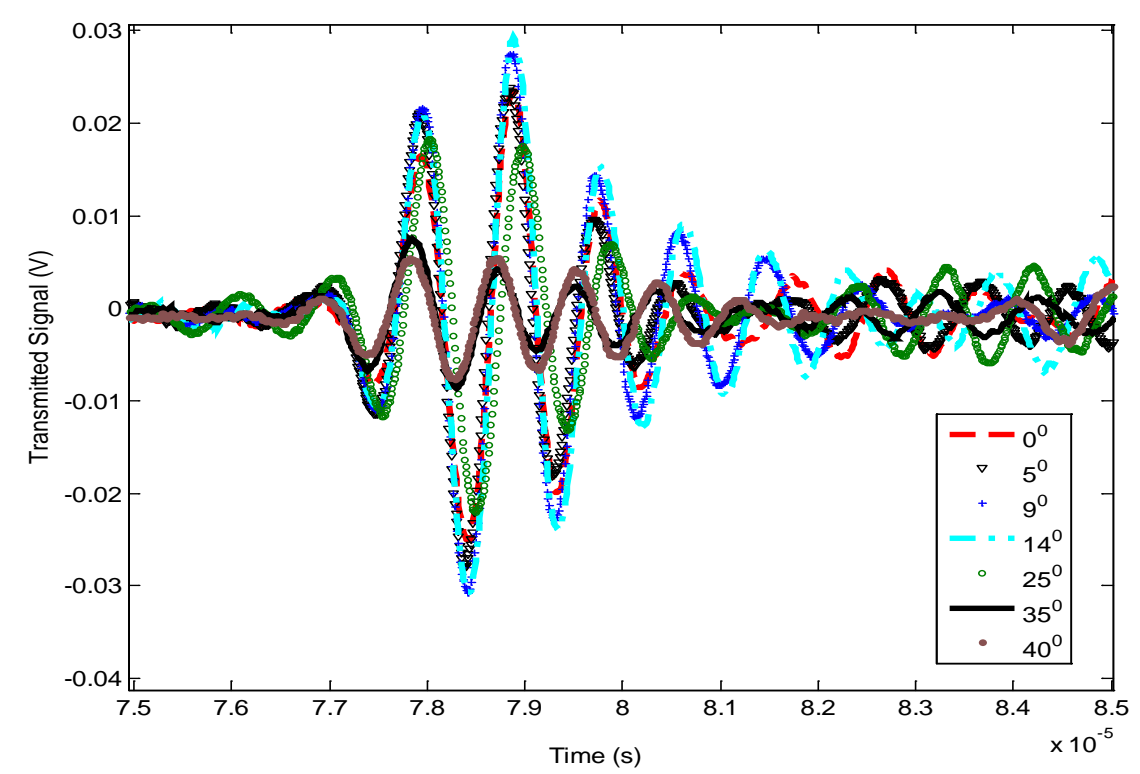

Figure 4: Comparison of measured transmitted waveforms through porous ceramic versus time for different angles of propagation.
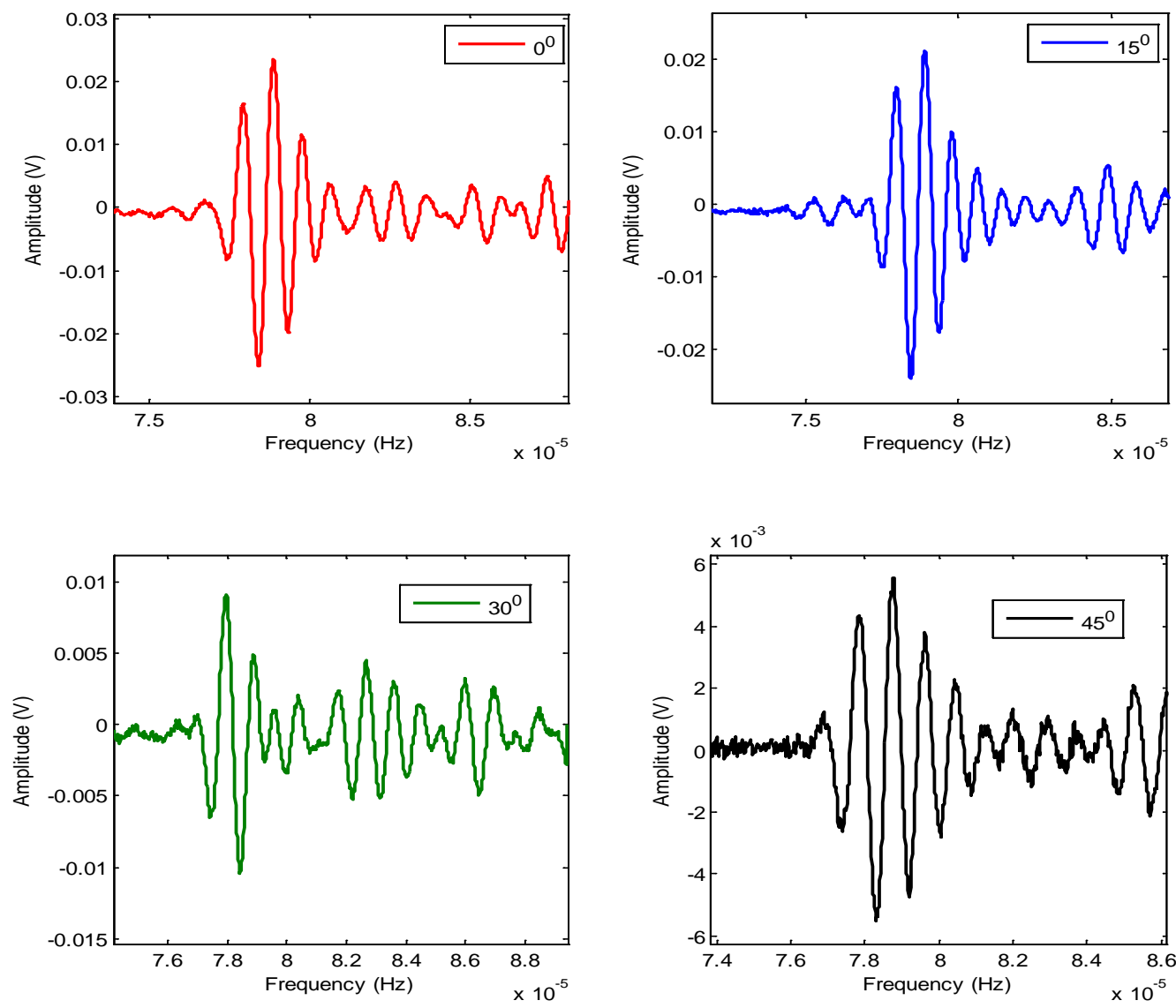

Figure 5: Measured transmitted waveforms through porous ceramic versus time at $0^{\circ}, 15^{\circ}, 30^{\circ}$, and $45^{\circ}$. 


\section{Theory}

A rigid porous sample of length $L$ is subjected to an incident ultrasonic wave in fluid (water), $P^{i}$. Part of incident wave is reflected back into the fluid, $P^{r}$, while other part is transmitted through the sample, $P^{t}$. Fellah et al. [8] have presented an analytical model in order to describe the viscous interaction between fluid and a porous elastic structure. The Fourier transform of the transmitted field is given by Fellah et al. [8] as:

$$
P_{3}(x, \omega)=\tilde{T}(\omega) \exp \left(-j \omega \frac{(x-L)}{c_{0}}\right) \varphi(\omega), \quad x \geq L
$$

where $\varphi(\omega)$ is the Fourier transform of the incident field $\left(P^{i}(t)\right), \tilde{T}(\omega)$ is the Fourier transform of the transmission kernel, $\omega$ is the angular frequency of motion, $c_{0}$ is the speed of sound in fluid, and $L$ is the thickness of the material. A more detailed consideration of the transformed field can be found in the paper by Fellah et al. [8]. The transmission coefficient $T(\omega)$, which is the Fourier transform of $\tilde{T}$, is given in the Appendix.

Aygün et al. [15] have introduced a transverse anisotropy into Biot-Allard model by allowing angle-and-porosity dependent tortuosity, and angle-dependent elasticity. A heuristic form for porosity- and angle- dependent tortuosity is proposed by Aygün et al. [15] as:

$$
\alpha_{\infty}=1-r\left(1-\frac{1}{\phi}\right)+k \cos ^{2}(\theta)
$$

where $\phi$ is the porosity, $\theta$ is the variable between $0^{\circ}$ and $90^{\circ}, r$ and $k$ can be considered adjustable. A range of possible values of $r$ and $k$ have been found by comparing predictions of equation (2) for $\theta$ $=0^{\circ}$ and $90^{\circ}$ respectively with values deduced from air-filled replicas of known porosity [16] Values of $r$ and $k$ are found by solving the resulting simultaneous equations.

To predict transmission through an anisotropic poroelastic sample it is necessary to allow for elastic anisotropy also. Williams [19] suggests that the dependence of skeletal frame modulus (Young's modulus, $E_{b}$, Bulk Modulus, $K_{b}$, and rigidity modulus, $\left.\mu_{b}\right)$ in terms of bone volume fraction $(1-\phi)$ and the Young's modulus of the solid material of the frame $\left(E_{s}\right)$ are given by $E_{b}=E_{s}(1-\phi)^{n}$, $K_{b}=E_{b} /\left(1-2 v_{b}\right)$, and $\mu_{b}=E_{b} /\left(1+2 v_{b}\right)$, respectively, where $v_{b}$ is the Poisson's ratio of frame, and the exponent $n$ varies from 1 to 3 according to Gibson [20], depending on the angle $(\theta)$ with respect to the dominant structural orientation according to $n=n_{1} \sin ^{2}(\theta)+n_{2} \cos ^{2}(\theta)$. Values of $n_{1}=1.23$ and $n_{2}=2.35$ are chosen by Lee et al. [5] to be consistent with the work of Williams [19]. 
The parameters used in the predictions are listed in Table 1. The elastic moduli of the porous ceramic made of clay has been taken to be equal to the elastic modulus of ceramic which is $59 \mathrm{GPa}$ and is higher than the elastic modulus of real bone which is $20 \mathrm{GPa}$ [19]. Assuming that the permeability of the bone is $5 \times 10^{-9} \mathrm{~m}^{3}[12]$, the permeability of ceramic has been taken to be $2^{2}$ times higher because the ceramic microstructures are larger than those of the actual bone microstructure by a factor of 2 in each direction. The assumed characteristics of the saturating fluid (water) are: density $\rho_{\mathrm{f}}=1000 \mathrm{~kg} / \mathrm{m}^{-3}$, viscosity $\eta=10^{-3} \mathrm{~kg} \mathrm{~ms}^{-1}$, speed of sound in water $c_{0}=1490$ $\mathrm{m} / \mathrm{s}$.

Table 1: Default input parameters for rigid porous ceramic

\begin{tabular}{ll}
\hline Parameters & Porous ceramic \\
\hline Density of ceramic, $\rho_{\mathrm{s}}$ & $1060 \mathrm{~kg} / \mathrm{m}^{3}$ \\
Young's modulus, $E_{\mathrm{s}}$ & $59 \mathrm{GPa}$ \\
Poisson's ratio of solid, $v_{\mathrm{s}}$ & 0.30 \\
Poisson's ratio of frame, $v_{\mathrm{b}}$ & 0.34 \\
Porosity, $\phi$ & 0.62 \\
Permeability, $k_{0}$ & $2 \times 10^{-8} \mathrm{~m}^{3}$ \\
Viscous characteristic length, $\Lambda$ & $799.5 \times 10^{-6} \mathrm{~m}$ \\
$r$ & 0.56 \\
$k,($ Eq. 2$)$ & 0.44 \\
Speed of sound in ceramic & $4000 \mathrm{~m} / \mathrm{s}$ \\
Thickness & $30 \mathrm{~mm}$
\end{tabular}

Only two parameters, Poisson's ratio of frame and viscous characteristic length, have adjusted in order to obtain the 'best-fit' at normal incidence. In particular, the predictions are very sensitive to the assumed values of viscous characteristic length. The 'best-fit' characteristic length values for the replicas listed in Table 1 are about 13 times those found for real bone i.e. between 5 and $10 \mu \mathrm{m}$ $[6,8]$.

\section{Comparisons between predictions and data}

Predicted transmission coefficient as a function of frequency for ceramic is shown in Figure 6. Predicted and measured transmitted waveforms in the Calcaneus and Femoral Head replicas at normal incidence are shown in Figures 7 and 8. There is reasonable agreement. It seems that only between 0.5 and $4 \%$ of the incident wave amplitude is transmitted through bone replicas at $1 \mathrm{MHz}$. Most of the ultrasonic wave is reflected back into the water. 


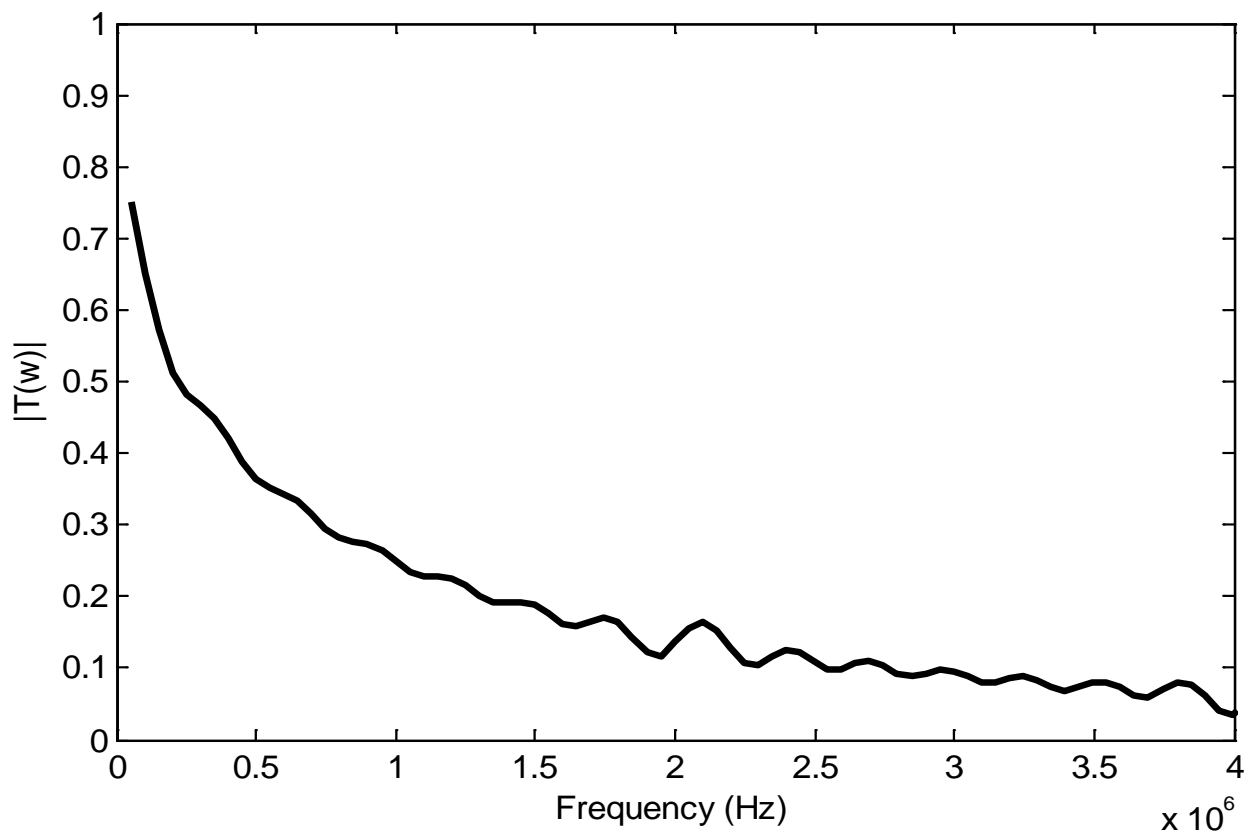

Figure 6: Numerical simulation of the transmission coefficient as a function of frequency at $1 \mathrm{MHz}$.

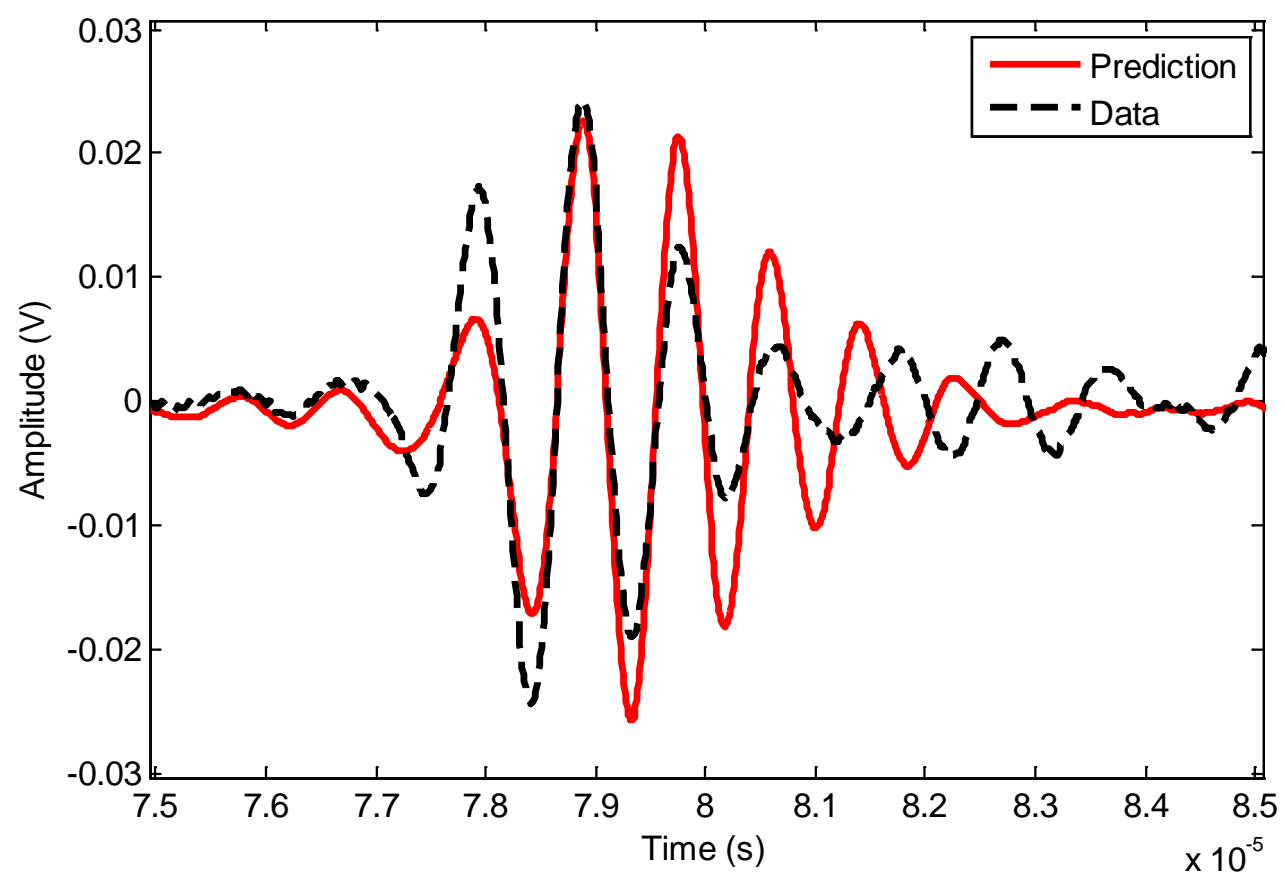

Figure 7: Predicted and measured transmitted waveforms in porous ceramic compared with data obtained at $1 \mathrm{MHz}$ and at $0^{\circ}$. 


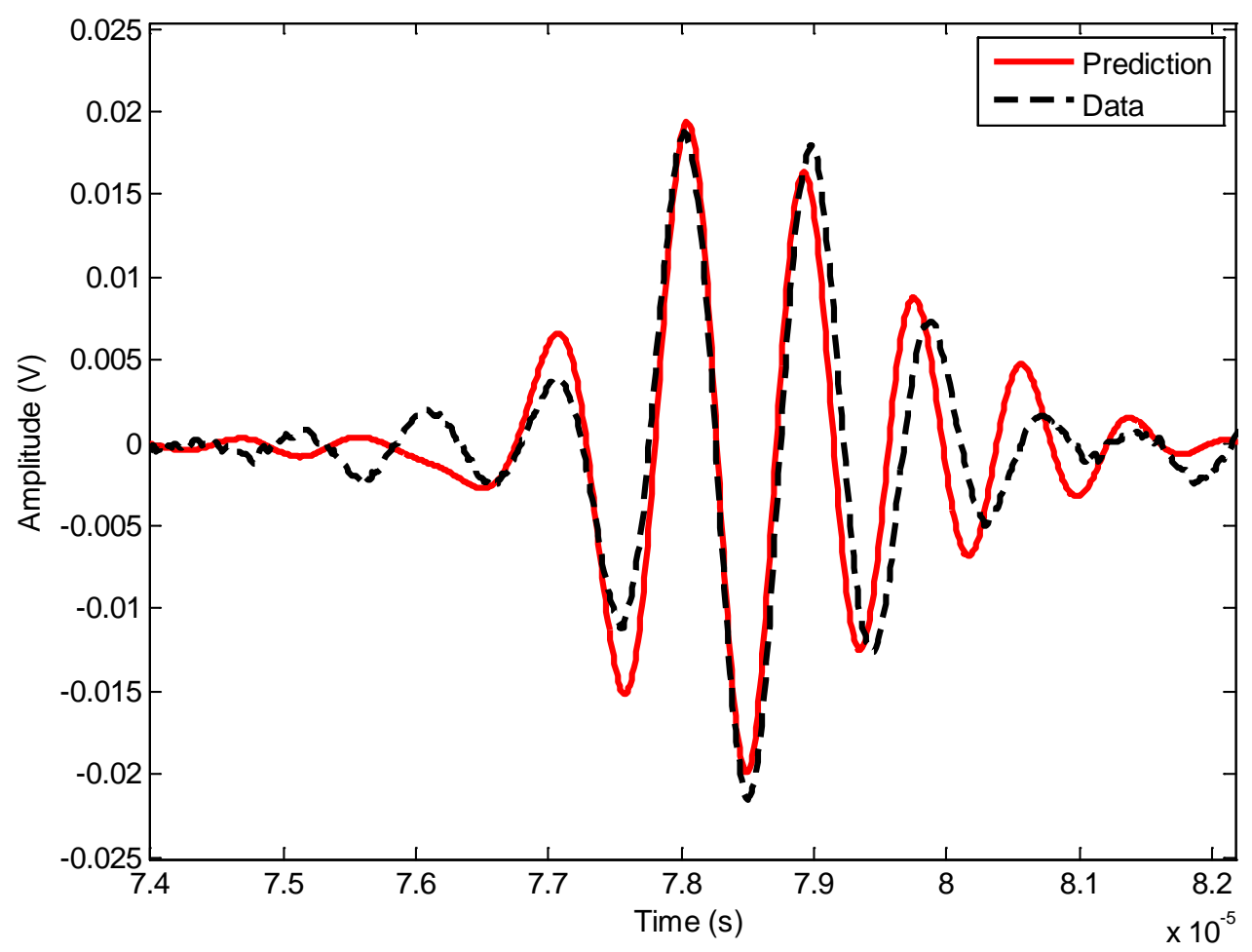

Figure 8: Measured and predicted transmitted waveforms through FRA bone replica versus time at $25^{\circ}$.

Generally measured and predicted transmitted waveforms are similar except for the initial parts of the transmitted waveforms which can be identified as the fast wave arrivals. The second and major parts of the transmitted waveforms can be identified as the slow wave contributions.

The predicted arrival time of the transmitted 'slow' ultrasonic waves changes when the angle of propagation is varied as a consequence of the angle dependence of tortuosity through equation (2).

\section{Conclusion and further work}

Predictions of a modified anisotropic Biot-Allard theory which neglecting scattering, have been compared with measurements of pulses centred on $1 \mathrm{MHz}$ transmitted through water saturated porous ceramics at normal and oblique angles. The predictions and data are in reasonable agreement. It seems that porous ceramics can be used to investigate the effects of Osteoporosis in cancellous bone on the mechanical and acoustical properties of the bone structure.

Further works need to be done on porous solid materials (ceramics) with different porosity values to investigate the Osteoporosis by using structural borne vibration. A low frequency $(<1000 \mathrm{~Hz})$ sinusoidal vibration should be applied to porous ceramics. This will cause a structural borne sound wave to propagate through the samples. The resulting vibration should be measured using either an ultrasound probe or an accelerometer. Based on these results, a hand held, low cost and portable 
device should be designed and developed to detect the Osteoporosis in the bone with the aim of improving early detection rates.

The chief debilitating consequence of osteoporosis is fracture, and early detection of the condition can allow interventions reducing the likelihood of occurrence [22]. As hip fracture invariably leads to hospital admission, early detection of the condition could significantly reduce the overall long term treatment costs by reducing admission to hospital [23]. Due to anticipated low costs of the product, it is anticipated that access to early screening would improve with increased overall take up by clinics compared to other technologies. The concept is for the device to have a simple interface, in order to be useable by health professionals without extensive training, to allow use in a variety of hospital departments and GP surgeries.

\section{Acknowledgement}

This work has been supported by Leverhulme Grant: F/00 181/N which provided for collaboration with the Laboratory of Acoustics and Thermal Physics at Leuven where the data reported here were obtained.

\section{References}

1. Johnell O., and Kanis J.A., 2006 An estimate of the worldwide prevalence and disability associated with osteoporotic fractures. Osteoporosis International 17: 1726-33.

2. British Orthopaedic Association, 2007 The care of patients with fragility fracture.

3. Burge R T, Worley D, and Johansen A, 2001 The cost of osteoporotic fractures in the UK: projections for 2000-2020. J. Medical Economics 4: 51-52.

4. Hughes E. R., Leighton T. G., White P. R., and Petley G. W., 2007 Investigation of an anisotropic tortuosity in a Biot model of ultrasonic propagation in cancellous bone. $J$. Acoust. Soc. Am. 121 568-574.

5. Lee K. I., and Choi M. J., 2007, Phase velocity and normalized broadband ultrasonic attenuation in Polyacetal cuboid bone-mimicking phantoms. J. Acoust. Soc. Am. 121 (6) EL263-EL269.

6. Sebaa N., Fellah Z., Fellah M., Ogam E., Wirgin A., Mitri F., Depollier C., and Lauriks W., 2006 Ultrasonic characterisation of human cancellous bone using the Biot theory: Inverse problem. J. Acoust. Soc. Am. 120 1816-1824. 
7. Lee K. I., and Yoon S. W., 2006 Comparison of acoustic characteristics predicted by Biot's theory and the modified Biot-Attenborough model in cancellous bone. J. Biomech. 39 364368.

8. Fellah Z. E. A., Chapelon J. Y., Berger S., Lauriks W., and Depollier C. 2004 Ultrasonic wave propagation in human cancellous bone: Application of Biot theory. J. Acoust. Soc. Am. 116 61-73.

9. Lee K. I., Roh H.-S., and Yoon S. W., 2003 Acoustic wave propagation in bovine cancellous bone: Application of the Modified Biot-Attenborough model. J. Acoust. Soc. Am. 114 2284-2293.

10. Hughes E. R., Leighton T. G., Petley G. W., and White P. R., 1999 Ultrasonic propagation in cancellous bone: A new stratified model. Ultrasound Med. Biol. 25 811-821.

11. Hosokawa A., and Otani T., 1997 Ultrasonic wave propagation in bovine cancellous bone. J. Acoust. Soc. Am.101 558-562.

12. McKelvie M. L., and Palmer S. B., 1991 The interaction of ultrasound with cancellous bone. Phys. Med. Biol. 36 1331-1340.

13. Biot M. A., 1956 Theory of propagation of elastic waves in a fluid saturated porous solid, I. Low frequency range. J. Acoust. Soc. Am. 28 168-1178.

14. Biot M. A., 1956 Theory of propagation of elastic waves in a fluid saturated porous solid, II. High frequency range. J. Acoust. Soc. Am. 28 179-191.

15. Aygün H., Attenborough K., Postema M., Lauriks W., and Langton C. M., 2009, Predictions of angle dependent tortuosity and elasticity effects on sound propagation in cancellous bone. J. Acoust. Soc. Am. 126 (6), 3286-3290.

16. Attenborough K., Qin Q., Fagan M. J., Shin H.-C., and Langton C. M., 2005 Measurements of tortuosity in stereolithographical bone replicas using audio-frequency pulses. J. Acous. Soc Am. 118 2779-2782.

17. Aygün H., Attenborough K., Lauriks W., and Langton C. M., 2010, “Ultrasonic wave propagation in Stereo-lithographical bone replicas.” J. Acoust. Soc. Am. 127 (6), 3781 3789. 
18. Aygün H., Attenborough K., Lauriks W., Rubini P.A., and Langton C. M., 2011, "Wave propagation in stereo-lithographical (STL) bone replicas at oblique incidence.” Applied Acoustics. 72 (7) $458-463$.

19. Williams J., L., 1992 "Ultrasonic wave propagation in cancellous and cortical bone: predictions of some experimental results by Biot's Theory,” J. Acoust. Soc. Am. 92, 11061112.

20. Gibson L. J., 1985 The mechanical behavior of cancellous bone. J. Biomech. 18 317-328.

21. Tucci JR. 2006, “Importance of early diagnosis and treatment of osteoporosis to prevent fractures.” Am J Manag Care. May;12(7 Suppl):S181-90.

22. King A.B., Saag K.G., Burge R.T., Pisu M., Goel N., 2005, "Fracture Reduction Affects Medicare Economics (FRAME): impact of increased osteoporosis diagnosis and treatment.” Osteoporos Int. Dec;16 (12):1545-57. 


\section{Appendix: Basis for the prediction of the Transmission Coefficient}

The transmission coefficient $T(\omega)$ is given by Fellah et al. [5];

$\mathrm{T}(\omega)=\frac{\mathrm{j} \omega 2 \rho_{\mathrm{f}} c_{0} F_{4}(\omega)}{\left[\mathrm{j} \omega \rho_{\mathrm{f}} c_{0} F_{4}(\omega)\right]^{2}-\left[j \omega F_{3}(\omega)-1\right]^{2}}$

where

$F_{i}(\omega)=\left\{1+\phi\left[\Im_{i}(\omega)-1\right]\right\} x \sqrt{\lambda_{i}(\omega)} \frac{\Psi_{i}(\omega)}{\sinh \left(l \sqrt{\lambda_{i}(\omega)}\right)} \frac{2}{\Psi(\omega)}, \quad i=1,2$

$F_{3}(\omega)=\rho_{f} c_{0}\left\{F_{1}(\omega) \cosh \left[l \sqrt{\lambda_{1}(\omega)}\right]+F_{2}(\omega) \cosh \left[l \sqrt{\lambda_{2}(\omega)}\right]\right\}$

$F_{4}(\omega)=F_{1}(\omega)+F_{2}(\omega)$

The eigenvalues $\lambda_{1}(\omega)$ and $\lambda_{2}(\omega)$ are the squared complex wave numbers of the two compressional waves and are given by;

$\lambda_{1}(\omega)=\frac{1}{2}\left[-\tau_{1} \omega^{2}+\tau_{2}(j \omega)^{3 / 2}-\sqrt{\left(\tau_{1}^{2}-4 \tau_{3}\right) \omega^{4}+2\left(\tau_{1} \tau_{2}-2 \tau_{4}\right)(j \omega)^{7 / 2}+\tau_{2}^{2}(j \omega)^{3}}\right]$
$\lambda_{2}(\omega)=\frac{1}{2}\left[-\tau_{1} \omega^{2}+\tau_{2}(j \omega)^{3 / 2}+\sqrt{\left(\tau_{1}^{2}-4 \tau_{3}\right) \omega^{4}+2\left(\tau_{1} \tau_{2}-2 \tau_{4}\right)(j \omega)^{7 / 2}+\tau_{2}^{2}(j \omega)^{3}}\right]$

where

$\tau_{1}=R^{\prime} \rho_{11}+P^{\prime} \rho_{22}-2 Q^{\prime} \rho_{12}$

$\tau_{2}=2\left(R^{\prime}+P^{\prime}+2 Q^{\prime}\right)$

$\tau_{3}=\left(R^{\prime} P^{\prime}-Q^{\prime 2}\right)\left(\rho_{11} \rho_{22}-\rho_{12}^{2}\right)$ and

$\tau_{4}=A\left(R^{\prime} P^{\prime}-Q^{\prime 2}\right)\left(\rho_{11}+\rho_{22}-2 \rho_{12}\right)$

Coefficients $R^{\prime}, P^{\prime}$, and $Q^{\prime}$ are given by

$R^{\prime}=\frac{R}{P R-Q^{2}}, Q^{\prime}=\frac{Q}{P R-Q^{2}}$, and $P^{\prime}=\frac{P}{P R-Q^{2}}$

where $P, R$, and $Q$ are generalized elastic constants.

The eigenvectors $\mathfrak{I}_{1}(\omega)$ and $\mathfrak{I}_{2}(\omega)$ are given by; 
$\mathfrak{J}_{1}(\omega)=\frac{\left(2 \tau_{5}-\tau_{1}\right) \omega^{2}+\left(\tau_{2}-2 \tau_{6}\right)(j \omega)^{3 / 2}-\sqrt{\left(\tau_{1}^{2}-4 \tau_{3}\right) \omega^{4}+2\left(\tau_{1} \tau_{2}-2 \tau_{4}\right)(j \omega)^{7 / 2}+\tau_{2}^{2}(j \omega)^{3}}}{2\left[-\tau_{7} \omega^{2}-\tau_{6}(j \omega)^{3 / 2}\right]}$

$\mathfrak{I}_{2}(\omega)=\frac{\left(2 \tau_{5}-\tau_{1}\right) \omega^{2}+\left(\tau_{2}-2 \tau_{6}\right)(j \omega)^{3 / 2}+\sqrt{\left(\tau_{1}^{2}-4 \tau_{3}\right) \omega^{4}+2\left(\tau_{1} \tau_{2}-2 \tau_{4}\right)(j \omega)^{7 / 2}+\tau_{2}^{2}(j \omega)^{3}}}{2\left[-\tau_{7} \omega^{2}-\tau_{6}(j \omega)^{3 / 2}\right]}$

where

$\tau_{5}=\left(R^{\prime} \rho_{11}-Q^{\prime} \rho_{12}\right)$

$\tau_{6}=A\left(R^{\prime}+Q^{\prime}\right)$

$\tau_{7}=\left(R^{\prime} \rho_{12}-Q^{\prime} \rho_{22}\right)$

The coefficients $\Psi_{1}(\omega), \Psi_{2}(\omega)$ and $\Psi(\omega)$ are given by;

$\Psi_{1}(\omega)=\phi Z_{2}(\omega)-(1-\phi) Z_{4}(\omega)$

$\Psi_{2}(\omega)=(1-\phi) Z_{3}(\omega)-\phi Z_{1}(\omega)$

$\left.\Psi(\omega)=2\left[Z_{1}(\omega) Z_{4}(\omega)-Z_{2}(\omega) Z_{3} \omega\right)\right]$

and the coefficients $Z_{1}(\omega), Z_{2}(\omega), Z_{3}(\omega)$, and $Z_{4}(\omega)$ by

$Z_{1}(\omega)=\left[P+Q \widetilde{J}_{1}(\omega)\right] \lambda_{1}(\omega)$

$Z_{2}(\omega)=\left[P+Q \mathfrak{I}_{2}(\omega)\right] \lambda_{2}(\omega)$

$Z_{3}(\omega)=\left[Q+R \mathfrak{I}_{1}(\omega)\right] \lambda_{1}(\omega)$

$Z_{4}(\omega)=\left[Q+R \mathfrak{I}_{2}(\omega)\right] \lambda_{2}(\omega)$ 